\title{
A METHOD OF IMAGE PLANE MULTIPLEX HOLOGRAPHY
}

\author{
Hsuan CHEN *, E.N. LEITH and Y.S. CHENG \\ The University of Michigan, Ann Arbor, MI 48109, USA
}

Received 16 August 1983

\begin{abstract}
A method for making image plane multiplex holograms is described. These exhibit the full 3-D imagery of conventional multiplex holograms, but can be made without the use of coherent light. A similarity to lenticular photography is noted; this similarity is then utilized in devising methods for constructing image plane multiplex holograms.
\end{abstract}

Multiplex holography [1--6] and lenticular photography [7] are two related methods for producing three dimensional images. The holographic method requires coherent light for the making process, as well as spatial, but not temporal coherence in the viewing. Lenticular photography requires no coherence in either making or viewing.

It is interesting and worthwhile to consider ways in which the coherence requirements could be reduced in the multiplex method, either in the making or viewing steps, or both. It is not unreasonable to expect that this could be done in view of the rather similar results obtained in lenticular photography, without the need for coherence. We note that a technique using achromatization methods has already been described for making multiplex holograms in spatially coherent white light $[8,9]$. Our interest here is in the use of spatially incoherent (i.e., extended source) light.

We start with the consideration that the coherence requirements arise because of the holographic nature of the multiplex process, namely, that a diffraction pattern of the object is to be formed. The formation of one hologram is shown in fig. 1. The transparency $s$ is illuminated with a coherent light beam, and a lens concentrates the light so that all parts of the object make contributions to the slit aperture. The light falling on the slit can be considered as either the Fourier transform of $s$, or the Fresnel transform of

* On leave from Saginaw Valley State College, Michigan.

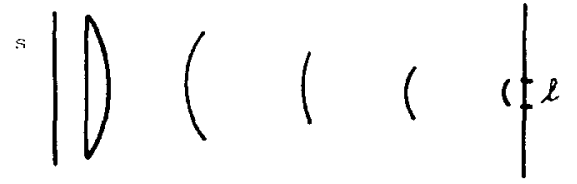

Fig. 1. The method of multiplex holography, showing the process of making one hologram. Reference beam, not shown, comes from below. $s$ is object transparency.

the product $s t_{a}$ where $t_{a}$ is the transmittance of the lens, being of the form $\exp \mathrm{j}(\pi / \lambda F)\left(x^{2}+y^{2}\right)$. In practice, the recording is done near, but not at, the focal plane of the lens, so that the zero spatial frequency component fills the aperture instead of being concentrated into an image point.

In the reconstruction process, the image forms at a distance $z$ from the hologram for the wavelength corresponding to the wavelength at which the hologram was made. Decreasing this distance reduces the coherence requirement for making the hologram. What effect would this have on the observed image? Would it appear closer, and thus exhibit less depth? The answer is no, since the depth at which the image forms has no relation to its perceived depth, which is determined by the convergence when two eyes observe the image through two different holograms that together constitute a stereo pair. Indeed, the image from a single hologram is found to be in almost equally good focus over a wide range of positions, from a distance up to less than $a \mathrm{~cm}$ from the hologram up to a distance of several meters. 
It would appear advantageous to reduce the object-hologram distance and indeed, reducing it to zero would result in an image plane hologram, for which the only coherence requirement in the making process is for producing interference between object and reference beam. This requirement can, with an appropriate interferometer, be nil. Here, we consider two possible forms an image plane multiplex hologram might have.

In the first form, we consider a process where the object transparency $s$ is close to the hologram, as in lenticular photography. Only a single element from each transparency (along the $x$, or horizontal direction) is stored on the hologram for each transparency. In this manner, $n$ pixel elements (from $n$ transparencies) are stored across the hologram aperture $l$, each having a width $l / n$. A reference beam $\exp \mathrm{j}\left[2 \pi f_{0} y\right.$ $\left.\left.+(\pi / \lambda F) x^{2}\right)\right]$ is introduced, thus forming a hologram

$1 / 2+s(x, y) \cos \left(2 \pi f_{0} y+\pi x^{2} / \lambda F\right)$.

The reference beam consists of the term exp $\mathrm{j} 2 \pi f_{0} y$, introduced in the vertical direction as in the usual multiplex process, the term quadratic in $x$ is a lens term, whose function we next explain, and $s(x, y)$ is the collection of pixel elements. Each pixel is recorded on a different portion of the zone plate structure defined by eq. (1) and then, when the hologram is illuminated with a line source of monochromatic light, each element is projected into a different direction. We can describe the process in a conceptually equivalent manner by considering the zone plate as a conventional lens with the various pixel elements recorded on the curved surface, as in fig. 2 . In the reconstruction process, a beam of light from a source narrow in the $x$ direction (the same type of source used in conventional multiplex holography) illuminat-

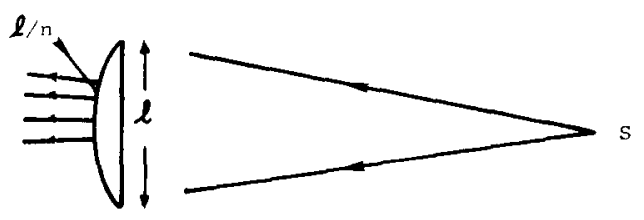

Fig. 2. The method of image plane multiplex holography, showing readout from one hologram. The conceptual refractive lens is shown instead of a zone plate lens. $S$ is source, $l$ is hologram diameter, $l / n$ is diameter of one pixel. es the hologram. Each wavelength is propagated in a different direction in the vertical plane. One wavelength component interacts with the hologram as shown in fig. 2 . The various pixel elements project diverging beams of initial size $l / n$ in various directions.

The device we have thus described can be constructed in various ways. One way, which is fairly simple, and also emphasizes the similarity to lenticular photography, is first to construct a lenticular photograph and then use it to form the multiplex hologram thus described. There is, however, a basic problem. We cannot simply make an image plane hologram of the lenticular photograph. A lenticular photograph is a complicated, 3-dimensional structure consisting of a picture (here called a multiple photograph) and a lens structure, located in different planes. One could of course make an image-plane hologram of the lenticular array and place behind it, a distance of one focal length, the multiple photograph. Such a structure is, however, a lenticular photograph using diffractive rather than refractive lenses, and is certainly not a multiplex hologram. We require, for a multiplex hologram, a structure that is not a spaced overlay of two separate transparencies.

There is an alternative procedure that leads to a true image-plane multiplex hologram. We proceed as in the basic method of lenticular photography, by making a multiple photograph for use with a lenticular array. Such a photographic plate, if placed at the focal plane of a lenticular array, would produce a conventional lenticular photograph. Suppose, however, we instead place the photographic plate in an imaging system, and the lenticular array in a second imaging system. The two systems are then placed in the two arms of an interferometer (fig. 3). The inter-

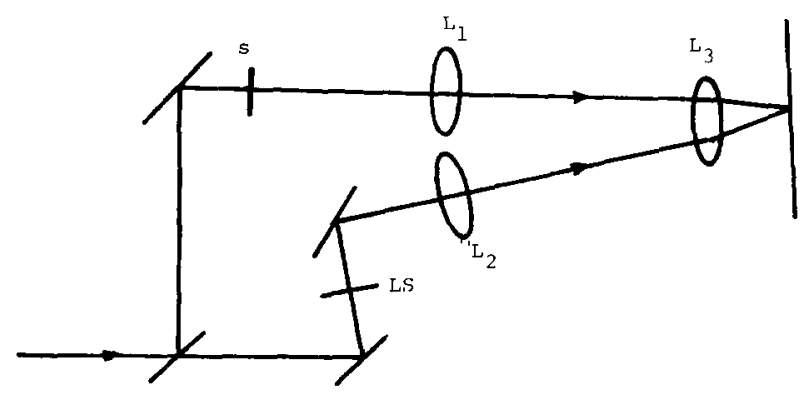

Fig. 3. Method of making image plane multiplex hologram, showing object $s$ and lenticular screen LS. Also shown are lenses $L_{1}-L_{3}$, mirror, and beam splitter. 
ferometer is a somewhat modified Mach-Zehnder that has been adjusted for extended source fringes. $L_{1}$ and $\mathrm{L}_{3}$ image $s$ to the recording plane while $\mathrm{L}_{2}$ and $\mathrm{L}_{3}$ image the lenticular $a$ array to the recording plane. An image plane hologram is thus recorded, as described by eq. (1), with the multiple photograph and lenticular structures being combined multiplicatively in a common plane.

An interesting variant of this arrangement is to place both the lenticular array and the multiple photograph in the same beam. To do this, one must overlay the lenticular array and the photograph, thereby forming a complete lenticular photograph. However, as already noted, we need them in a single plane, with the lens side of the array plane in contact with the photograph. Thus the lenticular plate is reversed and the photograph is in contact with the lenslet side of the lenticular array. This leads to the same result as when the two structures are in different beams. As a byproduct it suggests an alternative method of lenticular photography. By placing the photograph on the lenslet side of the array instead of on the focal plane side, we create a structure which can be viewed as a three dimensional image by back-illumination with a line source of light - either monochromatic or white. We found that this method works satisfactorily.

The multiplex hologram formed in the above way can be viewed like a conventional multiplex hologram. There remains the question of the image quality compared to the conventional multiplex hologram and the conventional lenticular photograph.

The basic resolution limitation in the multiplex process is the aperture effect. If the lenslets have a diameter $l$, then the resolution is $\Delta x=\lambda z / l$, where $z$ is the object-hologram distance, and $\lambda$ is the wavelength of light. Thus, it appears that a large aperture is better, but if too large, the image parallax occurs in jumps that are objectionally coarse. Collier et al., report an aperture size of $l=l_{\mathrm{e}} d_{\mathrm{o}} /\left(d_{\mathrm{o}}+d_{\mathrm{j}}\right)$ to be optimum, where $l_{\mathrm{e}}$ eye aperture, $d_{\mathrm{o}}$ a distance of viewer from hologram, and $d_{\mathrm{i}}=$ image-hologram distance.

For the lenticular case the situation is different. First, the resolution can never be better than $l$, since in the construction process, the resolution cells were of width $l$. In addition, a lenslet converts an object point into an approximately collimated beam that has an angular divergence of $\lambda / l$. Two beams from the same object point but from adjacent lenslets should not overlap, otherwise, the resolution is degraded by a factor 2. Also, if the two beams do not overlap, but both enter the pupil of the eye simultaneously, the resolution is again degraded. The proposed case, although related to both the lenticular and multiplex cases, has a resolution limitation more nearly related to the lenticular case. Again, the resolution cannot be better than $l$, since the lenticular process was used as an intermediate step in making the proposed image plane multiplex hologram. As with the multiplex, each image point produces a beam, and the various beams propagate both to the smaller aperture, $l / n$, and to the initial divergence of the beam. Again we require, for the best results, that beams from the same image point but on adjacent hologram elements do not overlap. Since the divergence is greater, we expect resolution to degrade with distance faster than in the lenticular case.

We analyze the resolution and the optimum hologram aperture for the proposed image-plane multiplex case. The resolution for the lenticular case is then found as a special case. For the analysis, we drop all considerations that are not fundamental, such as the resolution of the recording film, both for the lenticular and hologram stages, the loss of resolution in making the initial $n$ photographs, and aberrations in the lenslets. We thus obtain a result that is the upper limit of resolution. Since in practice the other factors are not negligible, the resolution achieved will tend to be poorer.

At the viewing position, a distance $d_{\mathrm{o}}$ from the hologram, we require that two beams from the same object point, but recorded on adjacent holograms, do not overlap. From fig. 4 , we find the separation of the beam centers to be

$x_{\mathrm{s}}=\left(d_{\mathrm{i}}+d_{\mathrm{o}}\right) l / d_{\mathrm{i}}$

and the width of each beam, from the basic geometry

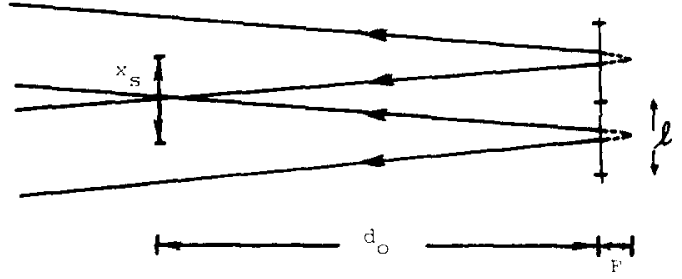

Fig. 4. Diagram for analysis, showing two beams from the same object point but from adjacent holograms. 


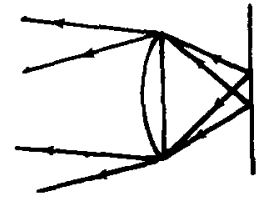

a

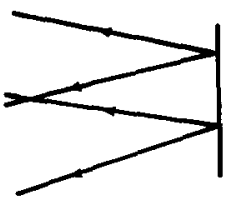

b

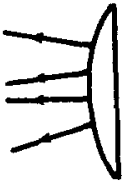

$c$

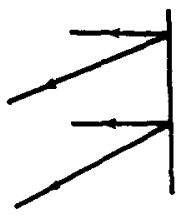

d

Fig. 5. Comparison of four cases: a. lenticular, b. multiplex, c. image plane multiplex, d. modified image plane multiplex.

of fig. 2 , is seen to be

$l\left(d_{\mathrm{o}}+F\right) / n F+\lambda d_{\mathrm{o}} n / l$,

where the first term is due simply to the divergence of the beams, without diffraction, and the second term is an additional factor, taking into account the diffraction effects.

Equating the two expressions gives

$l^{2}=\lambda n\left[\frac{1}{d_{\mathrm{i}}}+\frac{1}{d_{\mathrm{o}}}\left(1-\frac{1}{n}\right)-\frac{1}{n F}\right]^{-1}$

as a requirement on the hologram diameter. In the event the viewing distance $d_{\mathrm{o}}$ is much greater than the image depth, which normally is the case, eq. (4) becomes

$l^{2}=\lambda d_{\mathrm{i}} n /\left(1-d_{\mathrm{i}} / n F\right)$.

This equation is valid unless $x_{\mathrm{s}}$ is smaller than the eye aperture in which case the resolution is degraded by a factor $p$, where $p$ is the number of beams entering the eye from each object point.

A similar equation applies to lenticular photog. raphy. There are two differences, both manifest in eq. (3). First, $n=1$, and second, the beams do not diverge. Eq. (3) thus becomes $\lambda d_{\mathrm{o}} / l$, leading to the expression

$l^{2}=\frac{\lambda d_{\mathrm{i}}}{1+d_{\mathrm{i}} / d_{\mathrm{o}}} \approx \lambda d_{\mathrm{i}}$

as the lenticular photography counterpart of eqs. (4) or (5).

The proposed method is always less favorable than lenticular photography, but the differences are not necessarily large. For example, for $\lambda=0.0005 \mathrm{~mm}$, $d_{\mathrm{i}}=10 \mathrm{~mm}$ (a typical value for lenticular photography), $n=5$ and $F=4 \mathrm{~mm}$, we obtain values $l=0.07 \mathrm{~mm}$ for lenticular photography and $0.2 \mathrm{~mm}$ for the image plane multiplex method. The difference is in practice much less than the factor 3 indicated here, since other factors, such as lenslet aberrations and film resolution limitations give a value for the lenticular case far poorer than that calculated above.

A modification of the image plane multiplex method allows for an improvement in resolution, leading to the second of the two methods noted earlier. If the resolution cells were smeared to a width $l$, i.e., smeared over the entire hologram width, so that all $n$ elements overlap, but each, as before, directs by diffraction the incident light in a different direction, eq. (3) becomes

$x_{\mathrm{s}}=l\left(d_{\mathrm{o}}+F\right) / F+\lambda d_{\mathrm{o}} / l$

and eq. (5) is modified to

$l^{2}=\lambda d_{\mathrm{i}} /\left(1-d_{\mathrm{i}} / F\right)$.

With this modification, it would be desirable to let $F \rightarrow \infty$, since the focal power of the hologram no longer serves a purpose. Each overlapping pixel element is then on a constant spatial carrier that causes the incident beam to diffract into a plane wave that has divergence due to diffraction only; the divergence due to the sphericity of the beam is now eliminated. This is the most favorable case, using the smallest hologram size $l$ for a given depth $d_{\mathrm{i}}$. The expression for $l$ then becomes identical to that for integral photography. However, the overlapping of exposures results in reduced contrast.

The results are summarized in fig. 5 . In three of the four cases there is a basic similarity. The point spread function of the system is a planar or nearly planar wave of width $l$ emanating in a direction related to the position of the corresponding object point. Only the image plane multiplex case, in unmodified form, is different, where the point spread function is a divergent wave of width $l / n$.

Experimental results are shown in fig. 4 . An 


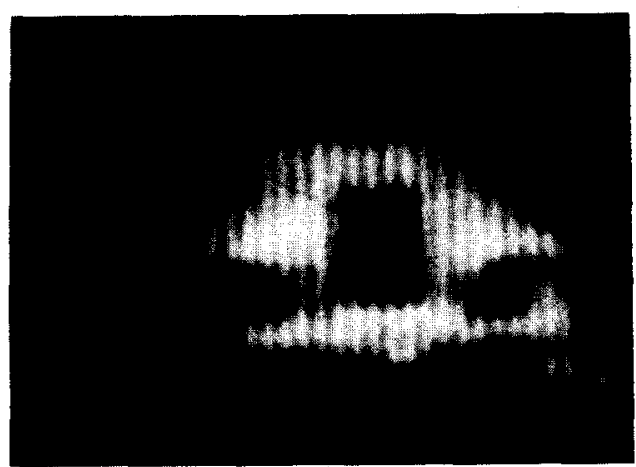

Fig. 6. Experimental result, showing one picture (of 30 pixel elements across horizontal direction) from an image plane multiplex hologram.

image plane multiplex hologram was made using the method described. A lenticular photograph was first made, consisting of 10 different views. This was converted into a multiplex hologram by interferometric means. In this case, the multiple photograph was placed in one beam and the lenticular sheet was placed in the other. A Mach-Zehnder interferometer was used, thus leading to a requirement for monochromaticity; the light source was therefore a laser, coherencespoiled by means of a rotating ground glass diffuser.
The resolution in the horizontal direction is just the lenslet diameter, about $0.5 \mathrm{~mm}$. This is the same resolution as would be produced by the same multiple photograph when converted into a conventional lenticular photograph by overlaying with a lenticular lens sheet.

This work was supported by the National Science Foundation (Grant NSF-G-ECS-8212472).

\section{References}

[1] S.A. Benton, J. Opt. Soc. Am. 50 (1969) 1545.

[2] J.T. McCrickerd and N. George, Appl. Phys. Lett. 12 (1968) 10.

[3] J.D. Redman, Proc. SPIE Seminar in depth holography (1968) p. 161.

[4] M.C. King, A.M. Noll and D.H. Berry, Appl. Optics 9 (1970) 471.

[5] D.J. DeBitetto, Appl. Optics 8 (1969) 1749.

[6] L. Cross, paper presented at annual meeting of the SPIE, San Diego, California, August 1977.

[7] T. Okoshi, Appl. Optics 10 (1971) 2284.

[8] E.N. Leith and G.J. Swanson, Appl. Optics 19 (1980) 638.

[9] G.J. Swanson, Appl. Optics 20 (1981) 4267. 